\title{
Una experiencia de significado y trascendencia didáctica en el proceso de lectura crítica
}

\author{
Edison Alberto Jiménez Valdés* \\ Edisson Argey Cadavid Cadavid ** \\ Sandra Alid Londoño Tohón*** \\ Recibido: 10 de mayo de 2017 \\ Enviado a pares evaluadores: 24 de mayo de 2017 \\ Aprobado por pares evaluadores: 30 de junio de 2017 \\ Aprobado por Comité Editorial: 14 de julio de 2017 \\ DOl: 10.22395/csye.v6n12a6
}

\section{RESUMEN}

El propósito fundamental del texto es presentar puntos de encuentro entre las áreas de Lengua Castellana y Filosofía, como producto hermenéutico de la propuesta investigativa que lleva por título: "La Lengua Castellana y la Filosofía: un encuentro de saberes que favorece la lectura crítica en los estudiantes del grado décimo en tres instituciones educativas del departamento de Antioquia", que permite trascender el discurso pedagógico con ideas, conceptos, procesos y acciones que visibilizan al maestro como sujeto. Es un acercamiento reflexivo, pedagógico y didáctico enfocado, desde la perspectiva curricular integradora de las áreas de Lengua Castellana y Filosofía en el grado décimo de educación media, a través del entendimiento y la comprensión de la propuesta La lectura, un acto de pensamiento y trascendencia. Esta propuesta es producto de la reflexión colectiva en la

investigación, sobre una serie de talleres dialógicos preparados por los docentes investigadores como material primario de intervención pedagógica. Entre los hallazgos más destacados se encuentra la integración de saberes en el proceso de lectura crítica, consolidando escenarios de transversalidad entre las áreas de filosofía y lengua castellana, comprobando la eficiencia del taller en el desarrollo de las competencias de los estudiantes y la planeación curricular de forma cooperativa por parte de los docentes. Desde esta dimensión se puede concluir que la lectura crítica, es un proceso que se debe promover desde la primera infancia como una experiencia cognitiva, sociocultural y creativa llena de sentido y significado dentro de un contexto.

Palabras clave: Lengua Castellana; Filosofía; integración curricular; lectura crítica; maestros.

\footnotetext{
* Magíster en Educación con énfasis en Didáctica de la Lectura y la Escritura, de la Universidad de Medellín. Docente en Educación Media de Lengua Castellana en el municipio antioqueño de Carolina del Príncipe. Correo electrónico: edisonjimenez02@gmail.com

** Magíster en Educación con énfasis en Didáctica de la Lectura y la Escritura, de la Universidad de Medellín. Directivo docente en el municipio antioqueño de Puerto Berrío. Correo electrónico: argeycadavid@gmail.com

${ }^{* * *}$ Magíster en Educación con énfasis en Didáctica de la Lectura y la Escritura, de la Universidad de Medellín. Docente en Educación Media de Lengua Castellana en el municipio antioqueño de Santa Rosa de Osos. Correo electrónico: hassanalid254@gmail.com
} 


\section{A meaning experience and a didactic significance in the critical reading process}

\section{ABSTRACT}

The main purpose of this text is to show meting points between Spanish and Philosophy areas, as a hermeneutic result of the research proposal under the name: "Spanish and Philosophy: a meeting of knowledge, which favors the critical reading in students of tenth level in three education institutions of Antioquia state," which allows going beyond the pedagogical discourse with ideas, concepts, and actions that make a teacher as a subject, visible. It is a reflexive, pedagogic and didactic approach focused, from an integrating curricular perspective, in Spanish and philosophy areas in Middle education tenth level students through understanding and comprehension of the proposal Reading, an act of thinking and significance. This proposal is a product of the collective reflection in the research, on a series of dialogic workshops prepared by teachersresearchers as a primary material of pedagogic intervention. Among the most outstanding findings, there is the integration of knowledge in the critical reading process, consolidating mainstreaming areas between philosophy and Spanish, thus verifying the efficiency of the workshop in the development of students' skills and the curricular planning in a cooperative way by teachers. From this dimension, it can be concluded that the critical reading is a process, which should be promoted from childhood as a cognitive, sociocultural, and creative experience full of meaning and sense within a context.

Keywords: Spanish, Philosophy, curricular integration, critical reading, teachers.

\section{Uma experiência de significado e transcendência didática no processo de leitura crítica}

\section{RESUMO}

O propósito fundamental deste texto é apresentar pontos de encontro entre as áreas de língua castelhana e filosofia, como produto hermenêutico da proposta de pesquisa sob o título "A língua castelhana e a filosofia: um encontro de saberes que favorece a leitura crítica nos estudantes do segundo ano do Ensino Médio em três instituições educativas do estado de Antioquia", que permite transcender o discurso pedagógico com ideias, conceitos, processos e ações que visibilizam o mestre como sujeito. É uma aproximação reflexiva, pedagógica e didática focada, sob a perspectiva curricular integradora das áreas de língua castelhana e filosofia, no segundo ano do Ensino Médio, por meio do entendimento e compreensão da proposta "A leitura, um ato de pensamento e transcendência". Essa proposta é produto da reflexão coletiva na pesquisa sobre oficinas dialógicas preparadas pelos docentes pesquisadores como material primário de intervenção pedagógica. Entre os achados mais destacados, encontra-se a integração de saberes no processo de leitura crítica, consolidando cenários de transversalidade entre as áreas de filosofia e língua castelhana, comprovando a eficiência da oficina no desenvolvimento das competências dos estudantes e do planejamento curricular de forma cooperativa por parte dos docentes. A partir dessa dimensão, pode-se concluir que a leitura crítica é um processo que deve ser promovido desde a primeira infância como uma experiência cognitiva, sociocultural e criativa, cheia de sentido e significado dentro de um contexto.

Palavras-chave: filosofia; integração curricular; língua castelhana; leitura crítica; professores. 


\section{Introducción}

La acción educativa es un universo amplio que permite ser pensado y comprendido desde diversas concepciones o perspectivas que posibilitan adentrarse a generar cuestionamientos, debates, reflexiones, conclusiones y propuestas. En este texto se pretende un acercamiento de forma vivencial y plurisignificativa al acto de la enseñanza de la lectura y su proceso de comprensión, enfatizando, desde una dimensión curricular y pedagógica, la importancia y trascendencia del empoderamiento del maestro como sujeto generador de un diálogo de saberes y experiencias que potencializan el trabajo en equipo con otros docentes, visiones y realidades que atraviesan el aprendizaje con un engranaje cognitivo. En este sentido: "el desarrollo de la transversalidad curricular como estrategia docente está unida de forma inexorable al desarrollo profesional del profesor, ya que este significa desarrollo, cambio, mejora, adecuación y crecimiento con relación al propio conocimiento y el contexto" (Fernández, 2004, p. 1).

Transversalidad curricular se refiere a una estrategia pedagógica, en la cual, a través de un eje generador o pregunta problematizadora se integran contenidos de diferentes áreas del conocimiento. Involucra que el proceso de enseñanza-aprendizaje sea más activo, donde estudiantes y docentes construyan nuevos conocimientos desde las necesidades y potencialidades que les ofrece su contexto, lo que supone reevaluar la práctica pedagógica e implementar nuevas estrategias, metodologías y reorganizar los contenidos, repensando el discurso pedagógico desde una perspectiva más integral.

En la propuesta investigativa denominada "La Lengua Castellana y la Filosofía: un encuentro de saberes que favorece la lectura crítica en los estudiantes del grado décimo de tres instituciones educativas del departamento de Antioquia": Cardenal Aníbal Muñoz Duque (Santa Rosa de Osos), Antonio Nariño (Puerto Berrio) y Presbítero Julio Tamayo (Carolina del Príncipe)", no solo busca involucrar a los estudiantes y docentes investigadores; sino también al grupo de educadores de las áreas de filosofía y lengua castellana de las Instituciones Educativas involucradas, con el fin de intercambiar experiencias y saberes; y, a través de esta relación de pares, implementar nuevas estrategias metodológicas que permitan la integración de estas dos asignaturas y lograr, de esta manera, el desarrollo de la lectura crítica en los educandos participantes en la investigación.

Estas acciones van orientadas a la planificación de acciones didácticas y curriculares que propicien el trabajo cooperativo entre docentes y estudiantes; en esta perspectiva:

Necesitamos romper las barreras existentes, y permitir el establecimiento de puentes que faciliten el trabajo en equipo, no solo al interior del área trabajada sino entre áreas afines. Ello permitiría una nueva cultura de trabajo basada en proyectos desde una perspectiva global (Fernández, 2004, p. 13). 
La integración curricular dentro del proceso educativo cumple un papel fundamental, ya que permite incrementar la comprensión de lo que se enseña, y es necesaria como un componente básico para construir la sociedad imaginada: más justa, más solidaria, más plural, una sociedad para vivir, donde la valoración por lo cotidiano, la investigación, el trabajo en equipo, la reflexión, la innovación, la búsqueda de una formación de seres humanos en interacción se haga en la práctica cotidiana, desarrollando habilidades de pensamiento que permitan a los estudiantes la capacidad de observar, criticar, analizar descubrir, inferir, predecir y transformar su propia realidad.

El proceso de integración curricular facilita el diálogo de saberes promoviendo la vinculación de todos los conocimientos de las áreas disciplinares, con un sentido de intencionalidad. Desde esta perspectiva es pertinente hacer referencia al concepto de integración curricular, señalado por Carlos E. Vasco:

No debe olvidarse que el propósito de la integración no es integrar disciplinas y áreas por el placer teórico y estético de lograr esa integración, sino propiciar en cada uno de los estudiantes, por medio de distintos tipos de estrategias la integración mental de los conocimientos entre sí, de estos con los valores, las actitudes, las actividades y la vida misma del estudiante. (Vasco, 1996, p. 33)

En este orden de ideas se pretende centrar la reflexión en una serie de estrategias que construyeron los docentes en su intervención, denominada "talleres dialógicos"; esta propuesta, más que alimentar el proceso investigativo, es el producto de la reflexión y contrucción colectiva de cada una de las comunidades de aprendizaje establecidas en cada una de las intituciones educativas; estos talleres combinan el sentir y el pensar de cada uno de los maestros, involucrando el discurso pedagógico como una construcción individual desde el ámbito didáctico y curricular. Cada docente se convierte en un sujeto versátil que proyecta en sus acciones en el aula una cantidad de categorias y conceptos que están ligados a la cultura, a las subjetividades que intervienen en el acto educativo y a cada una de las historias que se tejen en cada aula de clase.

\section{La Filosofía y la Lengua Castellana una alianza que genera empoderamiento en procesos de lectura crítica}

El ser humano, perceptivo y sensible por naturaleza, es también un ser de pensamiento y de lenguaje, que es capaz de construir imágenes e ideas de lo que experimenta, a la vez que comunica, comparte, lee, interpreta y hace juicios que le permiten construirse como sujeto y como ser social.

Leer es considerado como un acto cognitivo y sociocultural que va más allá de la mera descodificación de símbolos y de la configuración estructural de los mismos; se considera sociocultural en la medida que se representa desde las 
percepciones del sujeto; como acto cognitivo implica acciones como la reflexión, el pensamiento y la construcción de ideas; esas ideas tienden a hacerse complejas en la medida que van adhiriendo significaciones más complicadas; tal como lo afirma Ada Rodríguez (2007), "el lector deduce su significado en función de sus juicios, sus experiencias y su conocimiento del mundo" (Rodríguez, 2007, p. 40). De este modo el acto de leer está determinado por los significados que el ser humano ha construido en su experiencia y las ideas que, por ejemplo, pueda aportar la literatura.

Cuando leer está en función del pensamiento y de la reflexión puede hablarse de lectura crítica. Daniel J. Kurland (2003) define la lectura crítica como:

[...] una técnica que permite descubrir ideas e información dentro de un contenido escrito [...] hace referencia a una lectura cuidadosa, activa, reflexiva y analítica, y pone de antemano la idea de pensamiento crítico en la que es necesario reflexionar sobre la validez de lo que se ha leído teniendo como referencia el conocimiento y la comprensión del mundo que se observa (Kurland, 2003, p. 56).

El pensamiento se ha configurado como una herramienta en función del aprendizaje, pero también como una experiencia que permite leer la realidad circundante desde su complejidad, de modo tal que el ser humano puede leer su espacio vital y lo que sucede en él. Existe la pretensión de formar personas con pensamiento crítico, que vayan más allá de las cosas, que trasciendan al texto físico y lean la realidad de su entorno... la de quienes le rodean, la propia, y que esa capacidad albergue también la opción de ser problematizadores, creativos y transformadores. Es una finalidad en el sentido que proyecta un modelo de ser, de ciudadano, de estudiante que comprenda el sentido de las cosas, que sea capaz de trascender a la información, que se asuma con responsabilidad.

La idea de pensamiento crítico conduce, sin duda, al mundo de la Filosofía, donde la pregunta, el pensamiento, la interpretación y el conocimiento mismo tienen lugar. El mundo de la Filosofía parece esconderse en complejos laberintos retóricos y manifestaciones inconformes acerca de todo cuanto sea posible concebir, pensar, idear o poner de modo paralelo a la propia existencia del ser humano. Tratando de interpretar al sabio Protágoras, no sería posible afirmar la existencia de algo si no se dispusiera un límite, una medida, que viene siendo inevitablemente el ser humano, y su esencia observadora, curiosa, inquieta, creadora, imaginativa, escéptica, que lo conduce a preguntarse, a descubrir y hacerse una idea de todo.

El contexto social, la familia, el aula de clases, el espacio personal son cada uno un texto; sin embargo, si se pretende buscar códigos, símbolos, grafías en ellos como en otro texto no es muy satisfactorio el hallazgo, ya que los elementos que componen la realidad social de los seres humanos tienen otras formas donde 
son importantes las ideas, los pensamientos, las reflexiones y las acciones. $\mathrm{Si}$ bien estos no son posibles de leer como se adelanta la actividad de la lectura de textos escritos, sí alcanzan una singular relevancia porque permiten ubicar a la persona en su contexto, él como parte de lo que lee. Desde este punto, es posible identificar en la capacidad de leer la necesaria capacidad de comprender lo que ocurre en una situación específica del mundo que experimenta cada persona.

La lectura crítica es un medio, para llegar al pensamiento crítico, llegar a este punto justifica la propuesta de disponer de un estado dialógico en el que dos áreas de conocimiento se encuentran, Lengua Castellana y la Filosofía, defendiendo un marco para la reflexión sobre el mundo y la construcción crítica de conocimiento, desde un enfoque didáctico y pedagógico a partir del cual el maestro debe generar posibilidades para comprender la lectura crítica como una cultura de aprendizaje:

En el ámbito educativo el reto del trabajo en el aula, ha de ser formar lectores competentes como garantía para cultivar en los estudiantes la conciencia crítica y el espíritu científico, capaces de examinar, valorar, asociar, interpretar y argumentar con adecuación y pertinencia, la signifIcación que el discurso contiene (Serrano de Moreno, 2007, p. 67).

El hecho de llevar el proceso lector más allá de un texto y concebir la realidad como algo que se puede leer, sin duda, amplía la posibilidad de aprender; es por ello que en el aula de clases es posible hacer que encuentren los recursos propios del lenguaje con los del pensamiento, con el propósito de proyectar una lectura intencionada, reflexiva y orientada a leer para transformar

Desde este punto de vista, se pretende que el pensamiento y el lenguaje que, en la vida cotidiana de todo ser humano y de toda la colectividad están integrados en el acto de la comunicación, se apliquen de una forma significativa en el proceso de formación de lectores críticos en la educación media que prepara los estudiantes a una futura educación superior. La Lengua Castellana y la Filosofía deben ser una alianza significativa que potencie en los estudiantes la lectura y la escritura como actos de trascendencia y aprendizaje para la vida; es así como en ese ejercicio colectivo de pensarse y repensarse surge la propuesta de carácter metodológico con los docentes, incentivando un paradigma didáctico que contribuya a generar una conciencia pedagógica sobre la importancia de la concepción del control, la planeación, el seguimiento, la sistematización y la evaluación del nivel de lectura crítica.

En este sentido se trata de brindarles a los docentes una propuesta didáctica que surge de la búsqueda, la reflexión, la indagación y las concepciones didácticas con el fin de generar en las instituciones educativas comunidades de aprendizaje o pares académicos, en este caso de Lengua Castellana y Filosofía para didactizar lo que en cierto sentido es la metodología que desde el Icfes el Estado asume como objeto de medición; es una especie de familiarización e 
integración con los textos argumentativos de carácter filosófico que en algunas oportunidades resultan ajenos y apáticos a los jóvenes de la Modernidad; es una puesta en escena que pone en acción el pensamiento y el lenguaje como herramientas transversales y cognitivas en la experiencia de la lectura.

La ruta metodológica surge como producto pedagógico del proyecto de investigación La Lengua Castellana y la Filosofía: un encuentro de saberes que favorece la lectura crítica en los estudiantes del grado décimo de tres instituciones educativas del departamento de Antioquia, derivado de la reflexión originada por los pares académicos que conforman las comunidades de aprendizaje en cada una de las instituciones que intervienen en este proyecto investigativo; en este caso son los docentes de Lengua Castellana y Filosofía de grado décimo; su estructura consta de un paquete de doce (12) talleres dialógicos organizados en cuatro (4) unidades de trabajo, una (1) unidad para cada periodo académico del año lectivo; cada taller consta de un (1) texto argumentativo de carácter filosófico que pasa por cinco procedimientos o momentos didáctico que nutren la propuesta de intervención pedagógica titulada: "La lectura un acto de pensamiento y trascendencia", objeto de reflexión, entendimiento, comprensión y análisis en el presente artículo.

\section{La lectura crítica, una experiencia cognitiva y sociocultural, transversal en el aprendizaje}

La lectura debe ser concebida en el aula de clases más que como un simple proceso de descodificación del código lingüístico, como algo más que proponer un texto para interpretar y reproducir información; ella es más que dar cuenta de los datos explícitos de lo leído, de los personajes, de las ideas principales y secundarias. En este sentido:

Saber leer ya no sólo implica la descodificación léxico-sintáctica de oraciones, sino que implica un esfuerzo por la construcción de significado; todo ello, sobre la base de diversos elementos funcionales y contextuales que son relacionados por medio de la cognición del lector con el texto que se está leyendo (Parodi, 2010, p. 65).

Desde el ideal, la lectura debe ser una experiencia cognitiva y sociocultural que promueva nuevas perspectivas en el conocimiento del mundo; leer implica no solo entender y comprender lo leído, sino asumir una actitud crítica, reflexiva y propositiva según el tipo de texto que se presenta; es decir, reconocer, identificar, entender y comprender la estructura, intención o propósito comunicativo de cada parte del texto, para interpretarlo realmente sin perder su perspectiva; este punto de vista se comparte para posicionar la lectura y su proceso de comprensión como experiencias cognitivas que potencian el pensamiento, dimensionando el acto de leer como una construcción de significado desde la percepción de lo social y lo cultural: 
Respecto de la lectura, el nacimiento de la psicología cognitiva contribuyó a un cambio sustancial de los conceptos de lectura y comprensión: de ser considerada sólo como remodificación (del código de los fonemas al código de los grafemas), la lectura pasó a ser concebida como un conjunto complejo de procesos cognitivos equiparables. La comprensión, por su parte, pasó a distinguirse de la lectura; entendiéndose como la construcción de una representación coherente en la mente del lector, basada, tanto en la información contenida en el texto como en la memoria de dicho lector (Velásquez \& Peronard, 2006, p. 10)

No obstante, teniendo claro este proceso como la unidad de lo cognitivo con lo sociocultural, se hace necesario entender y comprender lo que se pretende con la propuesta "La lectura, un acto de pensamiento y trascendencia". Más que una simple planeación donde intervienen dos saberes, en este caso Lengua Castellana y Filosofía, se hace necesario reconocer que los niveles de lectura se deben abordar desde la primera infancia cuando el niño inicia su proceso metacognitivo para descubrir la realidad que alimenta su contexto como una experiencia de significado; bajo este entendido, el sentido de leer y comprender lo que se lee de forma consciente y permite hacer una caracterización de los niveles de lectura así:

El nivel de lectura literal que es un abreboca al mundo de la comprensión y de la interpretación, y que busca dar cuenta de qué dice el texto, en el que se trata de reproducir la información que el texto suministra de manera explícita y directa. En este mismo nivel existe una comprensión sencilla y básica del texto o imagen, en la cual el lector parafrasea la información encontrada. La lectura literal constituye la experiencia que predomina en el ámbito académico. Según Jurado \& Bustamante (1997, p. 92), en este nivel "el sujeto lee con la preocupación de la evaluación académica, y no tanto desde la evaluación textual, auténtica, presupuesta en todo lector analítico". Igualmente, Cassany (2006) considera este primer nivel, como la acción de leer las líneas, la superficie del texto.

El nivel de lectura inferencial exige mayor participación del lector quien, además, deberá inferir, deducir o concluir lo no explicitado en el texto; lo que se encuentra más allá de las líneas. Parafraseando a Jurado \& Guillermo1(997), en este nivel, la lectura es asumida como una auténtica práctica semiótica, de sentido y significado, un proceso flexible de exégesis y hermenéutica que requiere de un lector dialógico, capaz de leer, además del texto, el pretexto, el subtexto, el intertexto y el contexto. Leer de este modo prepara al estudiante para leer la vida.

El de lectura crítica se caracteriza por la alta complejidad para el lector, ya que contiene los dos niveles anteriores y le exige una práctica minuciosa a los diferentes tipos de textos que se le presenten al estudiante en este nivel de lectura; además, exige, valorar los argumentos incluidos en los textos para 
captar su sentido, diferenciar lo verdadero de lo falso, cuestionar posiciones y opiniones, entre otras acciones; es por ello que conduce necesariamente a la lectura y relectura, pero especialmente a la escritura, en la medida en que le otorga una estructura cohesiva y coherente a lo leído y debe ser justamente el punto ideal al que como docentes debemos conducir al estudiante.

Se añade, además, que la lectura crítica permitirá al lector ir asumiendo posturas estructuradas frente a los diferentes temas, es decir, trasciende lo meramente literal e inferencial y enjuicia el contenido, llegando inclusive a analizar el contexto de lo relatado, al involucrar en este ejercicio al autor, su entorno, la época y sus propias vivencias y realidad social. Aquí convergen las diferentes posturas, los diferentes conceptos, el proceso cognitivo y sociocultural; en este nivel se evidencia la capacidad del lector para no dejarse permear y antes bien argumentar lo que lo une o aleja de un texto.

\section{Talleres dialógicos: una estrategia pedagógica para la potenciación de las competencias de los estudiantes}

El término taller ha sido comúnmente empleado en el campo de la educación, y por la tradición con la cual lo han orientado muchos docentes ha generado en los estudiantes una actitud apática ante este término, relacionándolo directamente con un extenso y tedioso cuestionario sin sentido, donde resulta ser más importante la cantidad de la información que la calidad de la misma.

No obstante, el término también podría visualizarse como una experiencia en la cual se construye, se crea, se organizan piezas para formar un todo; en el campo pedagógico no se encuentra lejos de ser un espacio de construcción colectiva del conocimiento para obtener aprendizajes significativos.

El docente es la persona encargada del diseño del taller, y para esto debe tener muy en cuenta que este no puede aplicarse indistintamente en un contexto que en otro, porque los resultados serían completamente diversos; por ello, el educador debe ser un conocedor de sus estudiantes, sus intereses, sus dificultades y su contexto en general con el fin de que, efectivamente, el taller no solo motive a los estudiantes, sino que también se convierta para ellos en una construcción del aprendizaje, en un interactuar con el saber, y con sus pares académicos...

Frente a esta importante estrategia metodológica y didáctica, Melba Reyes describe lo siguiente:

El taller, como herramienta pedagógica permite desarrollar competencias aplicadas que permiten a los lectores fortalecer las capacidades que están estrechamente ligadas al hacer. 
También es asumido como una realidad integradora, compleja, reflexiva, en que se unen la teoría y la práctica como fuerza motriz del proceso pedagógico, orientando a una comunicación constante con la realidad social y como un equipo de trabajo altamente dialógico formado por docentes y estudiantes, en el cual cada uno es un miembro más del equipo y haces sus aportes específicos (Reyes, 1977).

Al respecto, José Carlos Núñez, en su artículo "Motivación, aprendizaje y rendimiento académico", señala la importancia de las capacidades, que corresponde al "poder" para desarrollar una actividad, y del "querer", que corresponde a la parte motivacional. Estos dos aspectos conjugados permiten el éxito en el acto educativo y la construcción de aprendizajes significativos en los estudiantes. Citando al autor,

El trabajo en esta línea de interacción cognición-motivación ha sufrido cambios sustanciales en consonancia con el modo de concebir el aprendizaje a lo largo de la historia de la investigación psicológica y educativa. De hecho, y a pesar de que la reflexión no es reciente, que ya Aristóteles sostenía que la inteligencia no era sólo conocimiento sino también la destreza de aplicar los conocimientos en la práctica, no ha sido hasta las últimas décadas cuando comenzamos a observar la cognición de un modo nuevo. No es tan importante develar la capacidad que uno posee como la forma en que utiliza esa capacidad: la destreza de aplicar los conocimientos en la práctica (Nuñéz, 2009, p. 8).

\section{"La lectura un acto de pensamiento y trascendencia": una propuesta que didactiza el proceso de lectura crítica}

El diálogo de saberes cargado de significados e intenciones como los que poseen los docentes desde sus experiencias cotidianas fortalece la construcción colectiva en los maestros; en este caso la propuesta: "La lectura un acto de pensamiento y trascendencia" es un aporte construido por docentes para docentes. Es una serie de talleres cada uno con 5 momentos didácticos: lectura, análisis, representación visual, dialógico y evaluación, los cuales se explican, a continuación, como un aporte valioso y significativo en la didáctica de la lectura.

Al iniciar el proceso lector, se hace una serie de actividades cognitivas desde la definición de lectura, el proceso de la misma y su clasificación que, según Solé (1992), incluye tres momentos: antes, durante y después de la lectura. En el primer sub-momento, el estudiante tiene la posibilidad de dar una mirada exploratoria al texto y generarse una idea previa del contenido del mismo, de acuerdo con sus experiencias, saberes, inquietudes, contexto, entre otros; es decir, realiza una serie de conexiones cognitivas que le permiten predecir el contenido del texto, propiciando con sus ideas iniciales un proceso dialógico con sus compañeros, que le permite ampliar su bagaje de conocimientos y comprender las posturas de las demás personas frente a una misma situación o texto. En este punto, el estudiante se encuentra motivado y con una serie de ideas sueltas para abordar la lectura del texto donde: 
El taller posibilita que los participantes propongan los tópicos del discurso, intercambien sus conocimientos, expresen sus intereses, dudas, temores, inquietudes y angustias, compartan sus experiencias, decidan cuándo inician y concluyen sus intervenciones, y manifiesten libremente sus opiniones y creencias sin la censura del docente (Coll, 1997, p. 22).

Asimismo, durante la lectura (segundo sub-momento), el educando pasa de realizar un diálogo con sus pares académicos a dialogar directamente con el texto y su autor, tratando de comprender la intención comunicativa que en él se encuentra. Para ello emplea distintas estrategias para identificar la idea principal, como es el caso del subrayado, de la formulación de preguntas al texto, en fin, todo el arsenal lingüístico y cognitivo del que dispone para lograr interactuar y comprender el texto.

Al culminar la lectura, el estudiante ha establecido nuevas conexiones de tipo cognitivo y cognoscitivo, interrelacionando sus conocimientos previos con la información suministrada por el texto. Ausubel (1983) plantea: "el aprendizaje del alumno depende de la estructura cognitiva previa que se relaciona con la nueva información [...] entenderse por "estructura cognitiva", al conjunto de conceptos, ideas que un individuo posee en un determinado campo del conocimiento, así como su organización" (p. 38).

Es por eso que cada lector comprende un texto de acuerdo con la realidad interior, con su experiencia previa, con su nivel de desarrollo cognitivo, su situación emocional. Pero esta relatividad no significa que no se puedan lograr niveles cada vez mayores de objetividad sobre el texto. La relatividad de la comprensión alude a que distintos lectores comprenden de forma diferente un mismo texto, lo cual se explica por la singularidad de los sujetos.

Es así como el docente, actuando como dinamizador, orienta para llevar a cabo procesos de comprensión, relectura y organización de las ideas a través de mapas conceptuales, gráficas, resúmenes, entre otros, de tal manera que el joven no solo comprenda el texto, sino que también se encuentre en capacidad de transmitir su comprensión frente al mismo.

Por su parte, en el momento 2 o momento de análisis, adquieren importancia los niveles de lectura: literal, inferencial y crítico se describieron anteriormente, y que son los evaluados en las pruebas de Estado (Icfes, a partir del año 2013) y los exámenes de ingreso a la educación superior.

Para desarrollar cada uno de estos niveles de lectura el educador puede emplear diversas estrategias pedagógicas, como, por ejemplo: para el nivel literal, las actividades deben enfocarse a la identificación de información en el texto, ideas principales, secundarias, palabras clave, entre otros; por su parte, para el 
nivel inferencial, encontrar las palabras desconocidas y generar su significado de acuerdo con el contexto; el docente también puede generar interrogantes que les permitan a los estudiantes reconocer la información implícita en el texto, utilizando parafraseo y deducciones; en el nivel crítico, el docente debe generar interrogantes que les posibiliten a los jóvenes ir más allá del texto, analizar, trasladar su contenido al contexto de la realidad y asumir posiciones frente a las ideas propuestas por el autor; para ello el lector debe recurrir a su arsenal de conocimientos, ideas, vivencias, en fin, a toda su competencia enciclopédica para ir más allá del significado del texto.

En este punto referido al análisis, cabe resaltar la importancia del proceso interdisciplinar llevado a cabo en el presente estudio entre las áreas de Lengua Castellana y Filosofía, pues en esta última área es común el análisis de textos argumentativos que, en primera instancia, resultan complejos y de difícil comprensión por parte del estudiante; sin embargo, a través de los talleres pedagógicos en donde los lectores adquieren herramientas lingüísticas y estrategias de comprensión textual, los educandos asumen con mayor motivación y tranquilidad los análisis de los discursos filosóficos, realizando deducciones, comparaciones, integrando su contenido a la realidad actual; y de este modo no solo se genera una mayor competencia crítica, sino que también se contribuye a la formación de buenos lectores en un contexto cada vez más dominado por la digitalización de la información y el mundo gráfico.

En el momento 3, o momento de representación visual, el educador orienta a los estudiantes sobre los distintos esquemas que pueden emplearse para representar la información como es el caso de líneas del tiempo, flujogramas, mapas conceptuales, cuadro sinópticos, y reconocer en ellos sus estructuras pragmáticas y semánticas, semejanzas y diferencias, entre otros; esto con el fin de que el estudiante se encuentre en capacidad de comprender lo leído, interactuar con esa nueva información y representarla a través de gráficos, de tal manera que dicha información perdure y se construya un aprendizaje significativo. Como lo plantea Ausubel (1983): "para aprender significativamente, el alumno tiene que manifestar una disposición para relacionar, de manera noarbitraria y no-literal (sustantiva), a su estructura cognitiva, los significados que capta con respecto a los materiales educativos, potencialmente significativos, del currículum" (Ausubel \& Novak, 1983, p. 38).

Continuando con la descripción de los momentos de los talleres, el momento 4 o dialógico encierra la esencia misma de la investigación; es un encuentro de saberes, un intercambio de opiniones; es el punto de encuentro entre la Filosofía y la Lengua Castellana, abordando los momentos anteriores, reflexionando y contextualizando el texto analizado; asimismo, en este momento, el estudiante realiza su propio texto filosófico sobre su reflexión teniendo en cuenta criterios 
de: objetividad (razón), subjetividad (sensibilidad), preguntas o cuestiones filosóficas (mayéutica), capacidad de asombro desde su experiencia de lectura; la escritura que se solicita al estudiante es libre, creativa y crítica con coherencia y cohesión, sin perder el sentido global del texto abordado en el taller.

Para finalizar, el momento 5 o momento de evaluación busca categorizar cualitativa y cuantitativamente la actividad desde el punto de vista del desarrollo del taller como tal; asimismo la identificación del docente de los niveles de lectura desarrollados por sus estudiantes; teniendo en cuenta la importancia de la evaluación dentro de los procesos educativos no solo se lleva a cabo la heteroevaluación, sino también la autoevaluación, donde los educandos analizan su propio avance en lectura crítica tras el desarrollo del taller pedagógico, propiciando la autoevaluación de forma consciente de cada estudiante, sobre los procesos metacognitivos, permitiendo una proyección de sus fortalezas y debilidades.

En este momento, tiene lugar la aplicación de una prueba tipo Icfes con cuestionamientos como le expresa Mauricio Pérez (2003) en su texto leer y escribir en la escuela: "Un buen lector hoy no es aquel que asimila mucha información; es quien logra, además de comprender, extraer conclusiones no dichas de modo directo en el texto y avanzar hacia la toma de posición frente a la información" (Pérez, 2003, p. 9).

Los momentos descritos anteriormente permiten entrever una aplicación de la teoría de las inteligencias múltiples, pues la variedad de actividades y estrategias posibilita al estudiante aprender y comprender con facilidad según su propio estilo de aprendizaje: escucha, observa y realiza diagramas, lee, explica, entre otros, con lo cual se están teniendo en cuenta en el proceso de desarrollo de la lectura crítica los ritmos, intereses y capacidades de los educandos. En esta perspectiva: "el mejor camino para empezar a comprender la mente humana consiste en examinar sus distintas estructuras, sus inteligencias individuales, para que, a la postre, sepamos también cómo vincular tales inteligencias y aprovecharlas con propósitos constructivos" (Gardner, 1987, p. 20).

Otro factor importante a resaltar dentro de la intervención pedagógica a través de la aplicación de los talleres es la metodología fundamentada en el constructivismo, puesto que el estudiante es el que organiza, interactúa y construye su conocimiento, y el docente ejerce una función orientadora en el proceso; igualmente, es evidente la importancia de los saberes proximales donde los lectores comparten con sus compañeros sus experiencias e interpretaciones de lectura, conectando en cierto sentido los postulados de la teoría de Vigotsky (Castorina, 2004). 
El desarrollo de la lectura crítica en los estudiantes, sujetos del presente estudio, permite prepararlos para enfrentar las pruebas de Estado y de ingreso a la educación superior, y les brinda herramientas que los preparan para continuar por el camino de formación profesional y afrontar los retos que representa una universidad. De este modo, se contribuye a la formación de lectores competentes, capaces de interactuar y entablar un proceso dialógico con los autores; lectores que se cuestionan constantemente sobre la vida, la sociedad, entre otros, como hace un tiempo lo hacían los filósofos; y estos cuestionamientos les permiten buscar soluciones a las problemáticas y transformar su contexto.

Es importante tener en cuenta que la sociedad y el mundo laboral actual se moviliza a través de procesos globalizados, sistematizados, donde han adquirido gran importancia los medios escritos para llevar a cabo las comunicaciones; por lo tanto, siendo la educación la encargada de formar personas competentes en la sociedad, se requiere que los futuros profesionales desarrollen la competencia de lectura crítica, de tal manera que no solo se encuentren en capacidad de comprender la información, sino también, de elaborar discursos propositivos y argumentativos que generen un avance en su mundo laboral y social.

No obstante, dentro del proceso de enseñanza-aprendizaje, es necesario iniciar con el desarrollo de la lectura crítica en los estudiantes desde los primeros grados de escolaridad, buscando fortalecer esta competencia grado tras grado, teniendo en cuenta que no solo es responsabilidad del docente de Lengua Castellana, sino de toda la comunidad educativa, pues en cada una de las áreas se requiere análisis de información y generación de aprendizajes significativos, y, quien desarrolle la competencia de lectura crítica, se encontrará en capacidad de aprender distintas artes y ciencias, por lo cual el proceso interdisciplinar será mucho más eficiente.

\section{Interacción docente en el desarrollo de propuestas integradoras: Un desafío en las instituciones educativas}

Teniendo en cuenta que todo el acto investigativo de este proyecto surge de la situación problémica ya planteada, y para la cual se diseñó la respectiva ruta metodológica, la propuesta se fundamentó en el diseño y aplicación de una serie de talleres pedagógicos, y para ello se llevó a cabo un trabajo mancomunado con los docentes de Lengua Castellana y de Filosofía; allí se tuvo la oportunidad de intercambiar conocimientos, experiencias, estrategias pedagógicas que enriquecieron la labor educativa, apuntando todos a un mismo objetivo: el desarrollo de la lectura crítica en sus estudiantes desde sus respectivas asignaturas.

En las instituciones educativas se hace pertinente que los educadores trabajen en equipo hacia las mismas metas en educación; de hecho, desde el 
Ministerio de Educación Nacional (MEN), se propone el trabajo y evaluación con pares académicos, de tal manera que en el intercambio de saberes sean aprovechadas las fortalezas de cada docente. Si desde el grupo de educadores se potencia el trabajo en equipo, se logra no solo un aprendizaje significativo en los docentes y estudiantes, sino también, procesos transversales en el conocimiento, de tal manera que desde un mismo eje o pregunta problematizadora o cualquier otra estrategia, se dirijan los conocimientos y actividades de las diferentes asignaturas.

Los educadores de Filosofía y Lengua Castellana deben dar a leer a los estudiantes textos a los estudiantes que los inciten a razonar, teniendo presente que los textos pueden ayudar a producir pensamiento más elaborado desde el planteamiento de lecturas filosóficas; es una tarea que no se puede realizar en el aula de clases de forma fragmentada; debe ser una visión que genere una sinergia complementaria entre el lenguaje y los procesos de pensamiento.

Se lee en la cotidianidad; se sabe que la lectura hace pensar, y el docente en su práctica debe orientar a los jóvenes a que aprendan a leer y a pensar; en este proceso es muy importante la práctica de intercambio o de comunicación que abra espacios para las preguntas, donde se debe volver a pensar lo que se cree saber, convertir lo desconocido o conocido en un espacio donde sea posible la interrogación y donde la creatividad y la creación sean pilares del aprendizaje. La educación implica la relación del otro con el mundo de la vida y es así como la experiencia de sí se enriquece con la experiencia del otro; construyendo un encuentro significativo de saberes, la identidad se construye en situación de alteridad, en situación con el otro como lo expresa Mélich:

En los procesos de configuración de la identidad, el otro se asume como presencia, es inquietante, reveladora y enigmática. Esos procesos, a su vez, son profundamente narrativos pues "el" "yo" no es una substancia, no es algo anterior, dado ya previamente a las situaciones, sino que es la autoexperiencia de la situación que se vive hacia fuera (Mélich, 2009, p. 89).

La enseñanza es un aprendizaje continuo y sistémico que les permite a los docentes generar espacios de reflexión, búsqueda, análisis e interpretación de las realidades que circundan el aula de clases y la Institución Educativa; en este sentido los docentes deben reunirse constantemente e intercambiar diversos procesos donde el objeto es la revisión estructural de los planes de áreas, mallas curriculares y microcurrículos, y hallar en las dificultades oportunidades de aprendizaje que generen un diálogo de saberes para que tanto docentes como estudiantes fortalezcan la relación de pensamiento y lenguaje como vértices fundamentales y transversales, no solo para el trabajo académico, didáctico y pedagógico de las dos áreas, sino como insumos de transversales en el acto pedagógico. 
Frente al párrafo anterior, es importante aclarar que el intercambio de ideas entre docentes debe trascender más allá del papel, pues en la actualidad a partir de los sistemas de calidad, se ha evidenciado que el docente ha pasado de ser sujeto activo del aula a objeto pasivo del papel; es decir, se plantean reuniones con pares académicos y revisión de documentación y currículos para diligenciar una serie de informes que se han convertido más en una obligación que en un resultado de reflexión consiente del acto pedagógico. Por ello, la reunión entre colegas debe convertirse también en un taller, una construcción de conocimientos y aprendizaje significativo entre pares, generando nuevos insumos y estrategias para ser aplicadas en el aula de clases para fortalecer los procesos didácticos y pedagógicos en el trabajo con los estudiantes.

\section{Resultados: la intervención pedagógica, un aporte a la didáctica de la lectura}

Como estaba planteado desde la ruta metodológica del trabajo, los talleres realizados buscaban el afianzamiento o fortalecimiento de la lectura crítica a través del análisis de textos argumentativos, donde la reflexión y el encuentro de las propias ideas con las del colectivo de estudiantes les permitiera desarrollar aptitudes y actitudes para potenciar las competencias necesarias para el proceso de comprensión e interpretación textual. Así pues, el análisis que se describe a continuación tiene en cuenta la forma en que los talleres ejecutados contribuyeron a la consecución de este objetivo, a la luz de lo que se planteó con este trabajo.

En efecto, como instrumento de enseñanza y aprendizaje:

El taller se basa en el principio constructivista según el cual, el educando es el responsable último e insustituible de su propio proceso de aprendizaje, en cuanto el proceso de adquisición de conocimientos es algo personal e intransferible. El taller es un aprender haciendo en el que los conocimientos se adquieren a través de una práctica sobre un aspecto de la realidad, el abordaje tiene que ser -necesariamente-globalizante: "La realidad nunca se presenta fragmentada de acuerdo con la clasificación de las ciencias o la división de las disciplinas académicas, sino que todo está interrelacionado" (Ander, 1991, p. 63).

En primera instancia, algo que se logró identificar en las tres instituciones, a diferencia del alcance que se había tenido desde la prueba diagnóstica, fue cómo los diversos momentos en los que estaban divididos los talleres permitieron establecer espacios de reflexión e intercambio de ideas entre los estudiantes y con los docentes, a partir de los cuales se hizo posible identificar en ellos el grado de conocimientos previos que tienen frente a los temas, los conceptos propios de cada área que manejan y el uso que les dan (siendo más evidentes las fortalezas o dificultades que presentan al respecto), su bagaje cultural, e incluso algunos gustos e intereses respecto a los procesos de lectura que se desarrollan en el aula. Es claro que toda esta información nutre el proceso de afianzamiento 
de competencias en torno a la lectura crítica y, dada la misma metodología de los talleres, da lugar a un proceso formativo mucho más enriquecedor para el estudiante y los intereses de este proyecto.

En general, aunque en los primeros ejercicios se hacía necesario hacer una relectura del texto trabajado, a medida que se fue avanzando con el trabajo se logró que un porcentaje mayor de estudiantes lograra asumir una postura inicial desde donde se construía una mirada global del texto, pero que en el caso de los textos de carácter argumentativo mostraban dificultad para definir claramente la tesis y los argumentos de peso plasmados por el autor para desarrollarla. Aunque los estudiantes participan en las actividades antes, durante y después de la lectura, se evidencia carencia de conocimientos previos con relación a la temática del texto y su autor, en coherencia con el MEN; hablamos de significación en sentido amplio, entendiéndola como aquella dimensión que tiene que ver con los diferentes caminos con los cuales los humanos llenamos de significado y de sentido a los signos, es decir, diferentes procesos de construcción de sentidos y significados; esta dimensión tiene que ver con las formas como establecemos interacciones con otros humanos y también con procesos a través de los cuales nos vinculamos a la cultura y sus saberes (Ministerio de Educación Nacional, 1998, p. 26).

Por ejemplo, ante la aparición de un término desconocido, muchos de los estudiantes tardaban en deducir su significado dentro del contexto del texto o incluso lo determinaban de forma errónea; y se supone que para el nivel donde se encuentran, este tipo de desempeños ya debe hacer parte de su cotidianidad en los procesos de lectura. Debe precisarse que este tipo de dificultad se hizo más recurrente en la Institución Antonio Nariño de Puerto Berrío, donde era necesaria la intervención y asesoría del docente para poder hacerlo de forma correcta.

Ahora bien, es necesario aclarar que esa conexión con lecturas de carácter filosófico hizo posible que, desde el intercambio de ideas, los estudiantes entraran en espacios de interpretación y razonamiento que, aunque deben fortalecerse en mayor medida, son una pieza clave en el desarrollo del pensamiento crítico. De igual forma, la necesidad de manejar conceptos básicos relacionados con el área de Filosofía y la poca familiarización que mostraron los estudiantes ante ellos, deja entrever que el bagaje conceptual con el que llegan a grado décimo no es el que se espera según lo planteado en los estándares y competencias definidas en la estructura curricular vigente, hallazgo que evidencia la necesidad de fortalecer este tipo de aspectos desde niveles de enseñanza inferiores.

Se observó gran dificultad para generar en los estudiantes estrategias cognitivas de forma autónoma que les permitan un buen entendimiento de lo 
argumentado por los autores, aunque a medida que se fue avanzando en los talleres pudo notarse una leve mejoría en lo referente a este tipo de desempeños.

También, al abordar la macro estructura de los textos argumentativos en este momento de la lectura, el gran problema para los estudiantes radica en identificar de forma clara y concreta la tesis expuesta por el autor, siendo necesario un constante acompañamiento y una serie de explicaciones o aclaraciones dadas por el docente, para que puedan llegar a delimitarla y asimilar qué argumentos son los que utiliza para su desarrollo dentro del texto y, con ello, asumir y exponer su propia postura o llegar a defenderla dentro de los debates que se plantearon.

Por otro lado, los espacios de debate y tertulia que se plantearon a los estudiantes, fueron asumidos con un alto grado de motivación por los estudiantes $\mathrm{y}$, aunque en algunas ocasiones no recurrieron a una exposición fluida de ideas, el intercambio de conceptos les llevó a aclarar ciertos elementos tratados en los textos trabajados, dentro de un ambiente donde ganaban, de forma progresiva, confianza para ser partícipes de estos espacios. Con esto, debe hacerse la reflexión que desde el rol del docente es muy importante movilizar y poner en práctica estrategias metodológicas y didácticas que desplacen el pensamiento en el proceso lector para que los estudiantes vayan más allá de los textos que leen y establezcan relaciones entre ellos (como medio para acceder a una postura crítica). Lo anterior se convierte en una interesante línea de acción que puede llegar a ser un punto de partida para profundizar los objetivos iniciales que se trazaron en este trabajo. En esta perspectiva la pregunta o el cuestionamiento al mismo texto, tanto desde el la Lengua Castellana como de la Filosofía toma una prioridad en el proceso cognitivo de comprensión del mismo texto:

\footnotetext{
El interrogante está en la base de la interacción didáctica, por lo tanto, la incorporación del diálogo como acción discursiva en el aula involucra el reconocimiento de los turnos, la necesidad de escuchar y de mantener la coherencia temática para el logro de los propósitos comunicativos, teniendo en cuenta no solamente las estructuras lingüísticas y las secuencias para construir relaciones lógicas, sino también las acciones que se desencadenan a través de la palabra (Molina, 1999, p. 72).
}

Se destaca como hallazgo importante el hecho en que se evidenció que la representación visual ayuda o fortalece los desempeños de los estudiantes en la descodificación de ideas, palabras y párrafos, contribuyendo a la construcción de sentido alrededor de la estructura de los textos que leen, categorizando de mejor manera algunas ideas que en los otros momentos no se hicieron evidentes. Aunque en ocasiones no hubo una jerarquización óptima de los conceptos, se logra que realicen con facilidad mapas conceptuales y mapas mentales, llegando a esquematizar muchas de las ideas plasmadas por los autores. También, este tipo de representaciones se convierten en herramientas cognitivas que permiten, desde la integración y articulación de las dos áreas, que los estudiantes 
construyan una visión más perceptible de la estructura de los argumentos que ya se aclararon y socializaron en momentos anteriores. Así la comprensión del texto es una experiencia social que permite generar múltiples percepciones del significado; en este sentido, la comprensión del texto entendida es como un proceso mental intencionado en el que el sujeto lector construye una interpretación de la información textual, basada en las pistas presentes en el texto y su conocimiento previo, para elaborar una interpretación, en la cual el lector utiliza una amplia gama de estrategias lectoras dada la diversidad de problemas a resolver, entre los que se cuentan: léxicos, sintácticos, semánticos, retóricos, pragmáticos y socioculturales.

\section{Conclusiones}

En las áreas de Lengua Castellana y Filosofía, se vivenciaron momentos valiosos para la disertación y la interacción literaria con el sujeto, logrando desarrollar talleres dialógicos que generaron una reflexión pedagógica profunda y un enriquecimiento didáctico para cada uno de los educadores participantes en la construcción de herramientas didácticas y pedagógicas que no solo permiten la integración, sino que posibilitan la transformación de paradigmas curriculares y el empoderamiento de la lectura y su proceso de comprensión como herramienta que atraviesa el aprendizaje.

La aplicación de la propuesta de intervención: "La lectura un acto de pensamiento y trascendencia" en la población objeto de estudio transformó la concepción de los estudiantes frente al concepto de taller, los motivó y los hizo parte activa de la construcción del conocimiento, y nutrió en ellos de forma colectiva e individual los procesos cognitivos que interactúan en el análisis e interpretación de los textos argumentativos en un nivel crítico.

Lo anterior permite afirmar que un taller correctamente diseñado, articulado y proyectado más allá de un contenido, y dirigido por el docente como agente orientador, permite abrir las posibilidades de aprendizaje de los educandos, fortaleciendo sus deseos por aprender y el desarrollo progresivo de las competencias.

En la experiencia práctica, llevada a cabo a partir de la investigación titulada“ La Lengua Castellana y la Filosofía: un encuentro de saberes que favorece la lectura crítica en los estudiantes del grado décimo de tres instituciones educativas del departamento de Antioquia" fue significativo el avance en motivación por parte de los estudiantes, quienes transformaron su pensamiento y concepción frente a los talleres y los textos filosóficos, sobre los cuales pudieron comprender más fácilmente, generar mayores reflexiones y relacionarlos con la cotidianidad; a medida que se avanzaba en los talleres y los estudiantes se mostraban más interesados, la participación era mucho más activa y se consolidaba el trabajo en equipo como una estrategia metodológica para aprender del otro y con el otro. 
El taller, como fundamento de la propuesta de intervención pedagógica y de cada uno de los momentos que nutren la estructura epistémica de la misma, potencia, de forma significativa, el proceso didáctico y curricular de las áreas de Lengua Castellana y Filosofía, arroja unos resultados de cambio de actitud por parte de los docentes en la gestión curricular de planeación y evaluación en los procesos de comprensión de lectura, y posibilita una interacción de competencias y estándares que provocan una sinergia colectiva desde el discurso pedagógico y las diversas acciones que forman parte de la realidad de los sujetos y el colectivo que forman parte de este proyecto.

Aplicadas las diferentes actividades del taller se observó en los jóvenes un gran disfrute por estrategias nuevas dentro de las clases, pero nace la reflexión de que no se pueden dejar las rutinas propuestas, ya que solo la puesta en práctica de las mismas contribuye al logro de los resultados esperados. La orientación dinámica del taller contribuyó a la presentación de una secuencia coherente de contenidos, fortaleció el nivel de lectura crítica en los estudiantes, desarrolló una serie de estrategias tendentes a la vinculación efectiva de los participantes y le brindó a la comunidad estudiantil espacios de reflexión y participación en procesos de integración de áreas del saber.

La lectura crítica es un componente que debe desarrollarse desde todas las asignaturas en la educación formal, como un eje trasversal del currículo; por ello, los docentes deben transformar sus prácticas pedagógicas de aula desde una concepción cognitiva, y generar en los estudiantes aprendizajes significativos para su formación académica y su vida personal

\section{Referencias bibliográficas}

Ander, E. (1991). El taller una alternativa de renovación pedagógica. Buenos Aires: Magisterio del Río de la Plata.

Ausubel \& Novak, J. (1983). Psicología educativa: un punto de vista cognoscitivo. México: Trillas.

Cassany, D. (2006). Tras las líneas. Barcelona: Anagrama.

Castorina, J. (2004). Psicología, cultura y educación: perspectivas desde la obra de Vigotsky. Centro de publicaciones educativas y material didáctico. Buenos Aires, 2-10.

Coll, C. (1997). Constructivismo y educación escolar: siempre de lo mismo ni lo hacemos siempre desde la misma perspectiva epistemológica. Barcelona: Paidós.

Fernández Batanero, J. M. (2004). La transversalidad curricular en el contexto universitario: un puente entre el aprendizaje académico y el natural. Revista Fuentes 5, pp. 1-12. Universidad de Sevilla.

Gardner, H. (1987). Estructuras de la mente: La teoría de las inteligencias múltiples. México: Fondo de la Cultura Económica.

Jurado, F., \& Guillermo., B. (1997). Los procesos de la escritura. Bogotá: Magisterio. 
Kurland, D. J. (26 de Julio de 2003). EDUTEKA. (U. ICESI, Editor) Obtenido de http://eduteka.icesi. edu.co/modulos/1/163/184/1 (26 de Abril de 2017)

Mélich, J.-C. (2009). Antropología de la situación (una perspectiva narrativa). En Skliar, C. y Larrosa, J. (Comp.) Experiencia y alteridad en educación. Rosario, FLACSO-Homo Sapiens, pp. 79-95.

Ministerio de Educación Nacional. (1998). Lineamientos Curriculares de Lengua Castellana. Bogotá: MEN.

Molina, H. B. (1999). La narrativa dialógica de Juana Manuela Gorriti. Bogotá: Universidad nacional.

Núñez J. C. (2009). Motivación, aprendizaje y rendimiento académico. Obtenido de http://www. educacion.udc.es/grupos/gipdae/documentos/congreso/xcongreso/pdfs/cc/cc3.pdf (27 de Abril de 2017)

Parodi, G. (2010). Saber leer. México: Instituto Cervantes.

Pérez, M. (2003). Leer y escribir en la escuela: algunos escenarios pedagógicos y didácticos para la reflexión. Bogotá: Ministerio de Educación Nacional.

Reyes, M. (1977). El taller en el trabajo social. Taller de integración de teoría y práctica. Buenos Aires: Humanidades.

Rodríguez, A. N. (2007). Lectura crítica y escritura significativa: Acercamiento didáctico desde la lingüística. (U. P. Libertador, Ed.), Laurus, 13(25), pp. 241-262.

Serrano de Moreno, S. y. (2007). Competencias de lectura crítica. Una propuesta de reflexión para la teoría y la práctica. Bogotá: Universidad de los Andes- Facultad de Educación y Humanidades.

Solé, I. (1992). Estrategias de lectura. Barcelona: Grao.

Vasco, C. E. (1996). Filosofía de la educación. Madrid: OEI.

Velásquez, M., \& Peronard (2006). Guiones metodológicos para desarrollar estrategias de comprensión y producción de textos escritos. Santiago de Chile: Ediciones universitarias de Valparaíso.

\section{Referencias bibliográficas complementaria}

Aprendizaje significativo (2014). Recuperado el 20 de mayo de 2017, de https://jesusangelmeneses. wordpress.com/

Cano García, M. E. (s. f.). La evaluación por competencias en la educación superior. Revista de Currículum y Formación del Profesorado.

Daros, W. R. (20 de marzo de 2017). Problemática sobre la "subjetividad-objetividad". Obtenido de https://williamdaros.files.wordpress.com/2009/08/w-r-daros-problematica-de-la-subjetividad-objetividad.pdf

Santillana (2011). Los métodos de la Filosofía. ¿Cómo aprender a preguntar? En Santillana, Sistema Uno. Bogotá: Editorial Santillana S. A.

Trilce, V. T. (2003). El aprendizaje verbal significativo de Ausubel. Obtenido de http://www.redalyc. org/pdf/373/37302605.pdf (24 de Marzo de 2017)

Vilarnovo, a. (s. f.). Objetivo y subjetivo: hermenéutica de la ciencia. Obtenido el 25 de marzo de 2017, de Depósito Académico Digital Universidad de Navarra: http://dadun.unav.edu/bitstream/10171/684/4/4.\%20OBJETIVO\%20Y\%20SUBJETIVO,\%20HERMEN\%C3\%89UTICA\%20DE\%20 LA\%20CIENCIA,\%20ANTONIO\%20VILARNOVO.pdf 